Research Article

\title{
Knowledge, Attitudes and Practices of Computer Vision Syndrome among Medical Students in Goa
}

\author{
Jagadish A Cacodcar', Tanvi Poy Raiturcar ${ }^{2}$, Rea R C Fernandes ${ }^{3}$, Shikha R Dessai \\ Varda Sinai Kantak ${ }^{5}$, Rutuja Naik
}

${ }^{1}$ Professor and Head, Department of Preventive and Social Medicine, Goa Medical College and Hospital, Bambolim, Goa, India. ${ }^{2}$ Senior Resident, Department of Ophthalmology, Goa Medical College and Hospital, Bambolim, Goa, India.

3,4,5,6 MBBS Student, Goa Medical College and Hospital, Bambolim, Goa, India.

DOI: https://doi.org/10.24321/2455.7048.202102

I $\quad \mathbf{N} \quad \mathbf{F} \quad \mathbf{O}$

\section{Corresponding Author:}

Tanvi Poy Raiturcar, Department of Ophthalmology, Goa Medical College and Hospital, Bambolim, Goa, India.

E-mail Id:

tanvi1491@gmail.com

Orcid Id:

https://orcid.org/0000-0001-8910-9982

How to cite this article:

Cacodcar JA, Raiturcar TP, Fernandes RRC, Dessai SR, Kantak VS, Naik R. Knowledge, Attitudes and Practices of Computer Vision Syndrome among Medical Students in Goa. Epidem Int. 2021;6(1):9-14.

Date of Submission: 2021-02-18

Date of Acceptance: 2021-03-21

\section{$\begin{array}{lllllllllllll}\text { A } & \text { B } & \mathbf{S} & \mathbf{T} & \mathbf{R} & \mathbf{A} & \mathbf{C} & \mathbf{T}\end{array}$}

Introduction: There has been a tremendous increase in the use of computers and other screens by young adults in educational institutions for education, communication, and recreation. This can lead to computer vision syndrome. Computer vision syndrome includes a variety of symptoms faced by individuals who use computers for long hours every day. Most early symptoms are not recognized and the condition goes undiagnosed. Creating public awareness about the healthy use of computers is the need of the hour.

Aim: To study knowledge, attitudes and practices of computer vision syndrome among medical students in Goa.

\section{Methods:}

Settings and Design: Cross-sectional descriptive study.

Study Duration: 1 month (June 2020)

Statistical Analysis Tools Used: Simple percentages and proportions.

Result: It is seen that among participants who use digital devices for more than 6 hours, 39 (92.9\%) were symptomatic. 62 (57.4\%) participants experienced worsening of symptoms due to lockdown.

Conclusion: The present study revealed that more than three-fourths of the students complained of one or more symptoms of computer vision syndrome while working on the devices.

Keywords: Goa, KAP, Computer Vision Syndrome, Medical Students

\section{Introduction}

Computer vision syndrome encompasses a constellation of ocular and extraocular symptoms in a person who either habitually or compulsively uses computers, mobiles, laptops, or tablets for long periods. ${ }^{1}$ This screen overexposure can cause vision problems and discomfort. Whether using a phone, tablet, laptop, or computer monitor, there is a range of symptoms experienced by the user. Eye strain, dry eyes, burning, watering, irritation, blurred or double vision, shoulder and back pain, and headache are chief 
symptoms. This further leads to long term morbidities like refractive errors, musculoskeletal related complaints such as tingling and numbness of the fingers, cervical stiffness, and backache. Poor visual function is the principal disadvantage. It increases mental stress levels. These lead to reduced effective working hours and frequent absence from work. All these ultimately lead to reduced productivity.

It has been seen that there has been a tremendous increase in the use of screens by young adults in educational institutions as well as at home, for education, communication, and recreation. ${ }^{3,4,5}$ Study materials have increasingly been more digitized, and education has been mostly carried out through online classes during the pandemic.

However, very little research has been carried out on Indian users especially among college students regarding the effects of computer use on the eye and vision-related problems. ${ }^{1,2,6}$ Also since no such study has been conducted in Goa, hence there is a need to investigate the (i) knowledge, (ii) attitudes, (iii) prevalence, and practices among medical students in Goa regarding computer vision syndrome and to evaluate the association of various factors in computer use with the occurrence of symptoms. ${ }^{1}$

\section{Materials and Methods}

A cross-sectional descriptive study was conducted over a month (June 2020) at Goa Medical College and Hospital.

The participants included 108 registered 6th semester students of MBBS course of Goa Medical College, between the age group of $20-25$ years.

Ethical approval was obtained from Institutional Ethics Committee of Goa Medical College.

The data for the cross-sectional study pertaining to knowledge, attitudes and practices of computer vision syndrome among medical students in Goa was collected using a pretested structured questionnaire after obtaining informed consent from participants. Their responses were kept confidential.

Given the ongoing COVID-19 pandemic, to prevent the risk of contagion spread, the research questionnaires were sent digitally through WhatsApp or e-mail to the students consenting to participate in the study.

The questionnaire included questions regarding demographic details such as age and semester, questions regarding usage of digital devices and symptoms:- type of digital device used: phone/ laptop/ tablet/ PC/ others, duration of use and continuous/ interrupted usage, screen distance, ocular and extraocular symptoms, sleep disturbance, ophthalmic evaluation, COVID-19 lockdown effect of symptoms if any, study modality used and questions regarding awareness of computer vision syndrome such as the 20-20-20 rule, night mode, blue light filter usage and ideal screen viewing angle. Few of the questions were open-ended to collect reasonable data.

The collected data were entered into Microsoft Excel version 16.3 and were statistically analyzed using SPSS 22 Software. The data were expressed as simple percentages and proportions.

\section{Result}

Overall, 108 students participated in the study. The age range was between $20-25$ years.

All the participants i.e. 108 (100\%) used mobile phones, 66 (61.1\%) used laptops, 13 (12\%) used personal computers and $2(0.9 \%)$ watched televisions.

Most participants i.e. 62 (57.4\%) used devices for 2-6 hours, 42 (38.9\%) used them for more than 6hours and 4 (3.7\%) used them for less than 2 hours.

Only 21 (19.4\%) out of the 108 students viewed the devices continuously while a majority i.e. 87 (80.6\%) used digital devices intermittently.

A majority 101 (93.5\%) of the participants felt an increase in screen time as a result of lockdown due to COVID-19 whereas 7 (6.5\%) felt it remained the same.

Most participants i.e. 68 (63\%) viewed the screen at chest level, 41 (38\%) at neck level, and 23 (21.3\%) at waist level.

The frequency of distribution of symptoms in the study included 54 (50\%) eye strain/sore eyes, 10 (9.3\%) eye irritation, $23(21.3 \%)$ watering,7 (6.5\%) blurred vision,13 (12\%) slowness of focus change, 49 (45.4\%) headache, 37 (34.3\%) neck/back pain and 34 (31.5\%) difficulty in concentrating while 25 (23.1\%) study subjects experienced no symptoms.

As far as sleep disturbance was concerned 45 (41.7\%) had difficulty in initiating sleep, 13 (12\%) faced difficulty in maintenance of sleep and 35 (32.4\%) experienced daytime sleepiness (Figure 1).

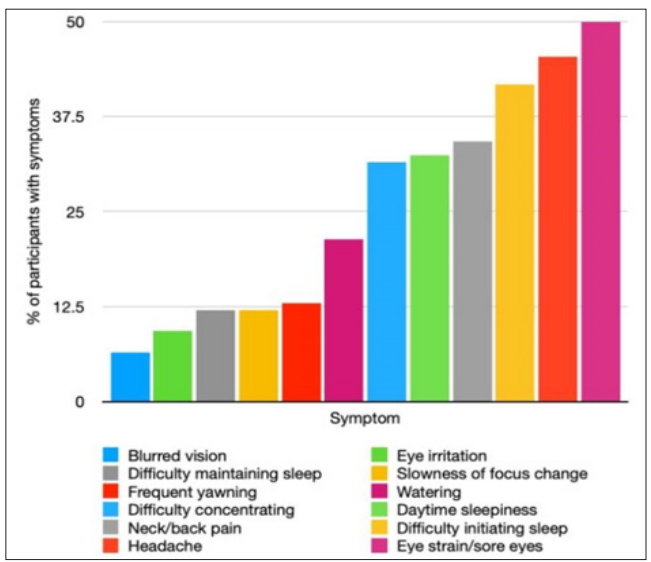

Figure I.Distribution of Participants based on Symptoms of CVS 
It was seen that $14(13 \%)$ participants experienced frequent yawning and 40 (37\%) had no sleep disturbance.

9 (8.3\%) participants had symptoms for days, 5 (4.6\%) had them for weeks, 41 (38\%) for months, 7 (6.5\%) for years while $46(42.6 \%)$ had no symptoms/were not sure. 62 (57.4\%) study subjects experienced worsening of symptoms due to lockdown whereas for 46 (42.6\%) participants, it remained the same.

For $44(40.7 \%)$ students, ophthalmic evaluation was done earlier whereas for 64 (59.3\%) students, it was never done. Out of 44 participants, 6 (54.5\%) were diagnosed to have dry eyes. Among these six, 2 (18.2\%) used lubricating ointment for treatment, 1 (1.9\%) used steroids and $3(27.3 \%)$ used systemic medications.

Among the total study subjects, 56 (51.9\%) had a refractive error and 52 (48.1\%) did not. Regarding the usage of contact lenses, 2 study subjects used them for months and 7 for some years. 1 (2.27\%) study subjects changed prescriptions a few days back, 20 (45.45\%) some months back, 8 (18.18\%) one year back, and 13 (29.54\%) more than one year back. Out of these, 38 (92.7\%) were comfortable with the change whereas $3(7.3 \%)$ were not comfortable.

$8(7.4 \%)$ used only books for studying, $8(7.4 \%)$ used only e-learning material and 92 (85.2\%) used both books and e-learning material.

68 (63\%) participants were aware of computer vision syndrome and 40 (37\%) are unaware. 83 (76.9\%) were aware of the causes of computer vision syndrome and 25 $(23.1 \%)$ were unaware.

$3(2.8 \%)$ participants viewed the screen at a distance of $15 \mathrm{~cm}, 91(84.3 \%)$ at $25 \mathrm{~cm}$ and $14(13 \%)$ at $35 \mathrm{~cm}$.

$70(64.8 \%)$ study subjects used digital applications in the dark mode and 38 (35.2\%) did not use the dark mode. 62 (57.4\%) study subjects used blue light filters and 46 (46.2\%) did not used them.

$7(6.5 \%)$ participants said that the ideal viewing angle is 10-20 degrees, 49 (45.4\%) said it was 20-30 degrees, 48 (48.44\%) said it was $30-40$ degrees and $4(3.7 \%)$ said it was more than 40 degrees. 28 (25.9\%) participants were aware of the management of CVS whereas 80 (74.1\%) were unaware. $24(22.2 \%)$ were aware of the $20-20-20$ rule and $84(77.8 \%)$ were unaware.

\section{Discussion}

Technological advances have made an impact on almost every aspect of our lives. Computers have revolutionized the workplace, accounting, designing, medical facilities, database management, experimental work, and the way daily tasks are executed. The advancement in the internet has opened up the option for users to access information highways available on the web and has enabled communication, due to which usage is increasing day by day. Personal computers and mobiles are now a necessity in offices, colleges, universities, and homes. Their use has led to easy access to information and an increase in efficiency in writing articles, and communicating with others. Millions of people including children, college students, and adults are using computers for prolonged hours nowadays. ${ }^{7}$

The prevalence of computer vision syndrome amongst the medical students was found to be $76.9 \%(83 / 108)$ with the medical students complaining of one or more computer vision syndrome manifestations as compared to $77.5 \%$ (359/463) found in another study conducted by Patil et al. ${ }^{1}$ Computer vision syndrome leads to sleep disruption, concentration problems, mental stress, daytime sleepiness and hampers cognitive and learning abilities, which in turn lowers academic performance. ${ }^{1}$

Out of 108 study subjects 52 (78.8\%) laptop users, 83 (76.9\%) phone users, 12 (66.7\%) tablet users, 7 (53.8\%) personal computer users, and $1(50 \%)$ television viewer suffered from symptoms related to computer vision syndrome.

The mean screen time was found to be 6.01 hours as compared to 3.5 hours found in another study by Reddy SC et al.5 The higher screen time in this study can be attributed to the online lectures and the lockdown due to which more time is spent on devices.

2 (50\%) study subjects who used devices for less than 2 hours were symptomatic, $42(67.7 \%)$ study subjects who used devices for 2-6 hours were symptomatic, while 39 (93\%) study subjects who used devices for more than 6 hours had symptoms.

62 (69.9\%) participants recognized worsening of symptoms due to the lockdown, while the rest 21 (30.1\%) seemed unaffected. All those using TV had sleep issues while 68 (63\%) participants using phone, 40 (78\%) laptop users, 6 (55.6\%) tablet users and 2 (46.2\%) television viewers had sleep-related issues.

$56(90.3 \%)$ among those who felt that the lockdown had worsened their symptoms also had sleep disturbances. 17 (80\%) among those who had a continuous pattern and 52 (59.8\%) among those with intermittent pattern developed sleep problems. $23(56 \%)$ among those with the screen at neck level, $42(61.8 \%)$ with the screen at chest level and $16(69.6 \%)$ with the screen at waist level had sleep disturbances.

Most of the participants were having these symptoms for the last few months. Those who had symptoms since the last few months agreed that the lockdown had a detrimental effect on their symptoms. 
Out of the 108 study subjects, $44(40.7 \%)$ had undergone an ophthalmic evaluation. $6(13.63 \%)$ study subjects had been diagnosed with dry eye. 2 (33.3\%) of those diagnosed with dry eye were prescribed ointment, 3 (50\%) were given systemic medications and $1(16.7 \%)$ received steroids.

$56(51.9 \%)$ participants had a refractive error, out of which $9(15.78 \%)$ used contact lenses, 2 (22.22\%) of whom had been using them for a few months and 7 (77.77\%) for more than 1 year.

Among the 2 participants using contact lenses for a few months, 1 (50\%) had foreign body sensation and 2(100\%) had worsening of symptoms during the lockdown. Among the 7 participants using lenses for more than a year, $3(42.85 \%)$ had eye strain, $1(14.28 \%)$ had watering, 2 $(28.57 \%)$ had blurred vision, and $3(42.85 \%)$ had worsening of symptoms during the lockdown.

$1(2.27 \%)$ had their last change of prescription a few days before the study, 20(45.45\%) a few months before, 8 (18.18\%) one year back, and 13 (29.54\%) more than a year ago.

38 (92.7\%) among those who last changed their prescription were comfortable with the change, while $3(7.3 \%)$ were not comfortable.

Out of the 3 study subjects uncomfortable with the change, 2 (66.66\%) had eye strain, 1 (33.33\%) had watering, 1 (33.33\%) had blurred vision and 1 (33.33\%) had difficulty in refocusing.

33 (58.92\%) students with refractive errors had sleep disturbance. 22 (39.28\%) had no sleep-related issues. Out of those with sleep disturbance, 23 (69.69\%) had difficulty in initiating, 7 (21.21\%) had difficulty in maintenance, 17 (51.51\%) had sleepiness during daytime/lectures, and 6 (18.18\%) experienced frequent yawning.

Out of the 2 participants using contact lenses for less than 1 year, 1 (50\%) had symptoms for months and 1 (50\%) for more than 1 year. Among the 7 students using lenses for more than 1 year, 4 (57.14\%) had symptoms for less than 1 year, and 3 (42.85\%) had no symptoms.

Out of the total participants, $8(7.40 \%)$ used books, 8 (7.40\%) used e-learning and 92 (85.18\%) used both for studying. Sleep disturbance was seen in $4(50 \%)$ of those who used books, 5 (62.5\%) who used e-learning and 60 (65.21\%) who used both as modes of studying.

This implies that sleep latency, sleep duration, habitual sleep efficiency, sleep disturbances, and daytime dysfunction are affected significantly by the usage of digital devices. ${ }^{1}$

It was seen that 68 (62.96\%) participants were aware and 40 (37.04\%) are unaware of computer vision syndrome. This is in contrast to the study which found that the students who had good knowledge about computer vision syndrome were only $22.46 \% .{ }^{1}$ This may be due to an increase in awareness due to social media. Out of $68(62.96 \%)$ students who were aware, 43 (63.23\%) used dark mode for screen viewing.

It was seen that $83(76.9 \%)$ participants were aware of the causes of computer vision syndrome which is similar to the study where it was found that $87 \%$ of people have knowledge of its causes. ${ }^{1}$

Among the study subjects who were aware of computer vision syndrome, 42 (61.76\%) had sleep disturbance. Out of the 40 who were unaware 29 (72.5\%) had sleep disturbance.

$3(2.8 \%)$ participants viewed the screen at a distance of $15 \mathrm{~cm}, 91(84.25 \%)$ at $25 \mathrm{~cm}$ and $14(12.97 \%)$ at $35 \mathrm{~cm}$.

$2(66.67 \%)$ study subjects had eye strain at $15 \mathrm{~cm}$ distance, $46(50.54 \%)$ at $25 \mathrm{~cm}$ and $6(42.86 \%)$ at $35 \mathrm{~cm}$.

According to Jaschinski et al.5, keeping the computer screen at a distance of 35-40 inches away may allow the eyes to relax and may reduce eye strain.

Among the 8 participants who used books for studying $3(37.5 \%)$ had symptoms for months, $1(12.5 \%)$ for years and $3(37.5 \%)$ had no symptoms. Out of the 8 participants who used e-learning for studying 6 (75\%) had symptoms for months and 2 (25\%) had no symptoms. Among the 92 (85.18\%) participants who used both books and e-learning for studying 12 (13.04\%) had symptoms for days, 46 (50\%) for months, $6(6.52 \%)$ for years and 13 (14.13\%) had no symptoms.

Among the total participants, 62 (57.4\%) were using blue light filters. From these 35 (56.45\%) had eye strain and sore eyes, 16 (25.8\%) had watering, 6 (9.67\%) had blurred vision, 34 (54.84\%) had a headache and 46 (74.19\%) had sleep disturbances as symptoms. Similar to an earlier study by Reddy SC et al the use of this filter did not help the students in reducing the symptoms of Computer Vision Syndrome.5 Out of the $68(62.96 \%)$ participants who were aware of computer vision syndrome 37 (54.41\%) were using blue light filters.

Blue light emission of the screen of devices may affect sleep: light mediates the synchronization of mammalian circadian rhythms with environmental time by modulating retinal input to the circadian pacemaker - the suprachiasmatic nucleus (SCN) of the hypothalamus. ${ }^{1}$ The suprachiasmatic nucleus contains neurons exhibiting a circadian pattern of activity and regulating melatonin secretion by the pineal gland in response to the environmental light/dark cycle. ${ }^{1}$ Melatonin hormone released in dim light conditions is involved in the physiological control of sleep. ${ }^{1}$ The intrinsically photosensitive retinal ganglion cells (ipRGCs) play a major role in nonimage-forming photoreception like regulating circadian photic entrainment. ${ }^{1}$ The release of melatonin 
from the pineal gland is controlled by a retinohypothalamic tract pathway originating from these ipRGCs containing melanopsin (having a peak sensitivity of approximately 482 $\mathrm{nm}$, that is, longer wavelength blue-turquoise light). ${ }^{1}$ The bright light of a computer screen may suppress melatonin secretion and delay the onset of sleep. ${ }^{1}$ Blue light emitted by digital devices has also been implicated as a cause of digital eye strain. ${ }^{1}$

$7(6.48 \%)$ students were of the opinion that the ideal angle of viewing is between 10-20 degrees,49 (45.37\%) considered it to be $20-30$ degrees, 48 (44.44\%) considered it to be $30-40$ degrees and 4 (3.7\%) thought it to be more than 40 degrees, hence suggesting the majority was unaware of it. Among those who were aware of it, no one had complaints of neck/ back pain. The level of the computer screen should ideally be 20-25 degrees below eye level such that the individual has to slightly tilt his/ her head forward when viewing the center of the screen (Figure 2). ${ }^{5}$ In a study, it was reported that a higher proportion of subjects who had their computer screen at or above the eye level reported asthenopia.5Adjusting the computer monitor to a viewing angle of 150 lower than the horizontal level may reduce the musculoskeletal discomfort (neck pain and back pain) and visual discomfort which supports our study findings. ${ }^{5}$ In our study, there was a significant reduction in symptoms of CVS among students who were aware of the ideal viewing angle.

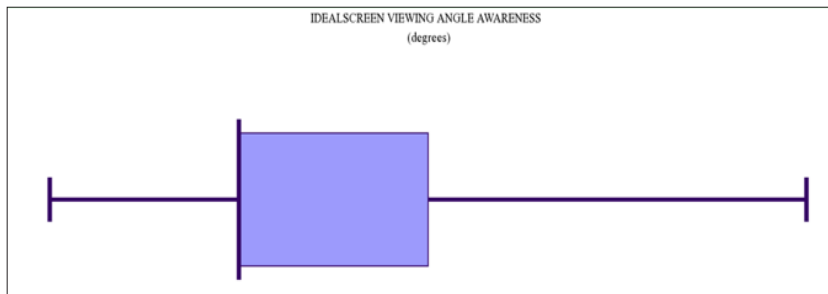

\section{$\begin{array}{lllllllll}10 & 15 & 20 & 25 & 30 & 35 & 40 & 45 & 50\end{array}$}

\section{Figure 2.Ideal Screen Viewing Angles}

Of all the participants, $27.77 \%$ adjusted screen brightness automatically, $44.44 \%$ manually and $27.77 \%$ used both methods. Light adjustment has a positive influence and it reduces computer vision syndrome. ${ }^{9}$

The screen lighting, contrast and brightness should be adjusted to the optimum before starting the work on the computer. $^{5}$

$24(22.22 \%)$ students were aware of the 20-20-20 rule, among whom, 11 (45.83\%) had eyestrain and sore eyes and $2(8.33 \%)$ had watering as symptoms whereas among the
$84(77.78 \%)$ participants who were unaware of it, majority $51.19 \%$ had eyestrain and sore eyes and $25 \%$ had watering.

Students who took frequent breaks were at a lower risk of developing symptoms of CVS as compared to those who did not take breaks. ${ }^{3}$

Taking short breaks of 5 min for every hour has been shown to decrease discomfort (eye and musculoskeletal) while not impeding productivity. ${ }^{3}$

Taking regular small breaks may relax the accommodation process of the eyes, thereby preventing eye strain. ${ }^{5}$

It will be easy to practice small breaks in between the work by following the rule of 20-20-20 i.e. after 20 minutes of computer use, one should look at something 20 feet away for 20 seconds. $^{5}$

Sleep disturbance was the most prevalent symptom second being sore eyes and eye strain, while the least prevalent was double vision. CVS is significantly associated with poor sleep quality in medical students. ${ }^{1}$ It has been reported that evening exposure to electromagnetic field influences sleep quality, attributing this to its probable modulatory effects on the circadian pacemaker; it may also result in altered cerebral blood flow and brain electrical activity. ${ }^{10}$ Exposure to mobile phone emissions at night time could have an effect on melatonin onset time. ${ }^{11}$ The electromagnetic field produced due to electrical appliances may play a significant role in influencing the circadian system because a substantial number of studies demonstrated the changes in melatonin and cortisol secretion (two major markers of the circadian system) as well as in sleep after exposition to these fields. ${ }^{12}$ In addition, physical discomfort such as muscle pain and headaches can be caused due to long hours engaged in mobiles, laptops, etc., thereby hampering sleep. ${ }^{13}$

Among those with sleep disturbances, sleep initiation difficulty was most the prevalent while sleep maintenance difficulty was the least prevalent.

Among those with CVS symptoms, the duration of symptoms since a few months was the most prevalent. This could be as a result of COVID-19 lockdown induced switching of learning and other things to online mode.

\section{Conclusion}

This study concludes that computer vision syndrome is a very common condition among undergraduate medical students. $83(76.9 \%)$ out of the 108 study subjects had one or more symptoms.

COVID-19 situation has worsened things as regards computer vision syndrome.

It is evident from our study that digital devices related health problems have now become a significant public 
health issue. There is a dire need to create public health awareness by organizing awareness lectures to sensitize the general public about the deleterious health effects of improper computer usage. The young generation should be addressed by organizing health awareness lectures at academic institutes to make the youth aware of health problems associated with prolonged improper use of digital devices.

\section{Source of Funding: None \\ Conflict of Interest: None \\ References}

1. Patil A, Bhavya, Chaudhury S, Srivastava S. Eyeing computer vision syndrome: Awareness, knowledge, and its impact on sleep quality among medical students. Ind Psychiatry J 2019;28(1):68-74.

2. Bali J, Neeraj N, Bali RT. Computer vision syndrome: $A$ review. J Clin Ophthalmol Res.2014;2(1):61-68.

3. Logaraj M, Madhupriya V, Hegde SK. Computer vision syndrome and associated factors among medical and engineering students in Chennai. Ann Med Health Sci Res 2014;4(2):179-85.

4. Payne KFB, Wharrad H, Watts K. Smartphone and medical related app use among medical students and junior doctors in the United Kingdom (UK): a regional survey. BMC Med Inform Decis Mak. 2012;12. Available from: https://s.docworkspace.com/d/AAFcMWZmqU2wdCE_badFA.

5. Reddy SC, Low CK, Lim YP, Low LL, Mardina F, Nursaleha MP. et al Computer vision syndrome: a study of knowledge and practices in university students. Nepal J Ophthalmol. 2013; 5 (102):161-168.

6. Rajeev A, Gupta A, Sharma M. Visual Fatigue and Computer Use Among College students. Rajeev, A Gupta, M Sharma - Indian Journal of Community Medicine. July -September, 2006 Jul-Sep;31(3).

7. Mohammed lqbal M, Ahmed El-Massry A, Mohammed Elagouz M, Hosam Elzembely H. Computer Vision Syndrome Survey among the Medical Students in Sohag University Hospital, Egypt. Ophthalmology Research: An International Journal. 2018;8(1): 1-8.

8. Khola Noreen K, Zunaira Batool Z, Tehreem Fatima T, Tahira Zamir T. Prevalence of Computer Vision Syndrome and Its Associated Risk Factors among Under Graduate Medical Students . Pak J Ophthalmol. 2016;32(3).

9. Firdous Jahan F, Zahid ul Islam Z, Muhammed Rafei M. Factors Leading to Computer Vision Syndrome in Medical Students: A Descriptive Analysis. International Journal of Public Health Research. 2018; 6(3): 78-82.

10. Huber R, Treyer V, Borbély AA, Schuderer J, Gottselig JM, Landolt HP, et alWerth E, Berthold T, Kuster N, Buck A, Achermann P. Electromagnetic fields, such as those from mobile phones, alter regional cerebral blood flow and sleep and waking EEG. J Sleep Res. 2002;11(4):289-95.

11. Wood AW, Loughran SP, Stough C. Does evening exposure to mobile phone radiation affect subsequent melatonin production? Int J Radiat Biol. 2006;82(2):69-76.

12. Lewczuk B, Redlarski G, Zak A, Ziółkowska N, PrzybylskaGornowicz B, Krawczuk M. Influence of electric, magnetic, and electromagnetic fields on the circadian system: Current stage of knowledge. Biomed Res Int. 2014;2014:169459.

13. Thomée S, Härenstam A, Hagberg M. Mobile phone use and stress, sleep disturbances, and symptoms of depression among young adults - A prospective cohort study. BMC Public Health. 2011;11:66.

14. Sanjeev Davey S, Anuradha Davey A. Assessment of smartphone addiction in Indian adolescents : A mixed method study by Systemic review and meta-analysis approach. Int J Prev Med. 2014;5(12):1500-1511. 